\title{
Solution speciation and implication on nucleation mechanism
}

\author{
Tonglei $\mathrm{Li}^{1}$ \\ ${ }^{1}$ Industrial \& Physical Pharmacy, Purdue University, West Lafayette, United States \\ E-mail: tonglei@purdue.edu
}

Deepening our understanding of solution chemistry and its impact on nucleation process is of critical importance for better control of production and product quality of crystalline materials in pharmaceutical, agrochemical, and fine chemical industries. Interactions among solute and solvent molecules dictate structural evolvement of self-assembled aggregates and nuclei, and influence crystallization kinetics. We have sought the relationship between nucleation mechanism and selfassociation of tolfenamic acid (TFA) in solution. In this study, we examined the nature of pre-nucleation solution aggregates of TFA in several possible distinct solvents and their impact on polymorphic nucleation through utilizing a combination of NMR and FTIR spectroscopies and density functional theory (DFT) calculations. Two distinct self-association pathways were identified of TFA in solution. In toluene, TFA forms hydrogen-bonded dimers via carboxylic acid, whereas in DMF and ethanol, the solutes hydrogen-bond with solvent molecules and develop weak self-association through aromatic stacking interactions. It was further discovered that carboxyl hydrogen-bonded dimers exist as both planar-like and twisted-like conformations in toluene. DFT calculations revealed that solute-solvent interactions in DMF and ethanol surpass those of TFA's carboxyl dimer formation; the stronger interaction between TFA and DMF leads to the formation of DMF solvate, while the crystal structures generated from ethanol bear only TFA molecules without solvent molecule present crystallographically. The structural connection between solution speciation and crystal structure(s) seems apparent in toluene and DMF. In ethanol, no direct similarity seems to exist, suggesting that ethanol molecules have to break off from solvated-TFA molecules during the nucleation process. The results nonetheless unveil a consistent picture where solute-solvent and solute-solute interactions mutually determine solution chemistry. Whether there is direct chemical linkage or not between the solution chemistry and resultant crystal structure, we do believe that the solution chemistry plays the critical role in determining the nucleation pathway leading to the crystal formation. In DMF and toluene, the solution species are carried out and preserved in the resulting crystal structures. In ethanol, the solution aggregates preserve the stacking association while the solvent molecules are repelled from the nuclei. In all, the pre-nucleation aggregates ultimately controlling the crystallization outcome.

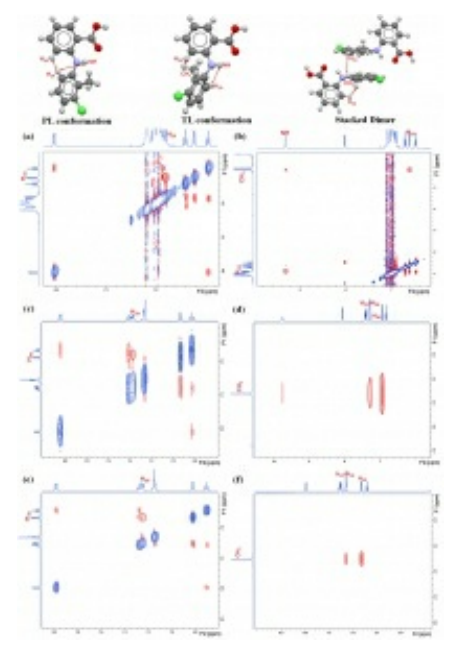

Keywords: Nucleation, solution chemistry, self-association 